\title{
Bidding Strategies in Austrian and German Balancing Power Auctions
}

\author{
Fabian Ocker, Karl-Martin Ehrhart† Marion Ott ${ }^{\ddagger}$
}

\author{
Article Type:
}

Advanced Review

\begin{abstract}
Balancing power markets involve complex procurement auction mechanisms that are challenging both to design for auctioneers and to take part in for suppliers. Recent empirical work indicates that auction results from Austria and Germany do not match game-theoretic predictions. Instead, suppliers adjust their bids to previous auctions results and do not reveal their actual costs within their bids. Therefore, this work focuses on bidding strategies of suppliers in the Austrian and German automatically-activated Frequency Restoration Reserve auctions. First, the operating principle of the auctions is analyzed and the cost and profit structures are illustrated. Then, a theoretic approach for the derivation of optimal bidding strategies is presented, that allows the integration of price expectations based on historic market data. We validate our approach by a numerical application of the bidder's calculus. Finally, our theoretic results are confronted with Austrian and German auction outcomes. We find evidence that the identified bidding strategies are applied by the suppliers.

This is the peer reviewed version of the following article: Ocker, F., Ehrhart, K.-M., Ott, M. (2018): Bidding Strategies in Austrian and German Balancing Power Auctions. WIREs Energy Environ. 2018;e303, which has been published in final form at https://doi.org/10.1002/wene.303. This article may be used for non-commercial purposes in accordance with Wiley Terms and Conditions for Use of Self-Archived Versions.
\end{abstract}

\footnotetext{
*Institute for Economics, Karlsruhe Institute of Technology (KIT), Germany, fabian.ocker@kit.edu

${ }^{\dagger}$ Institute for Economics, Karlsruhe Institute of Technology (KIT), Germany, ehrhart@kit.edu

${ }^{\ddagger}$ School of Business and Economics, RWTH Aachen University, Germany, marion.ott@rwth-aachen.de
} 


\section{GRAPHICAL TABLE OF CONTENTS}

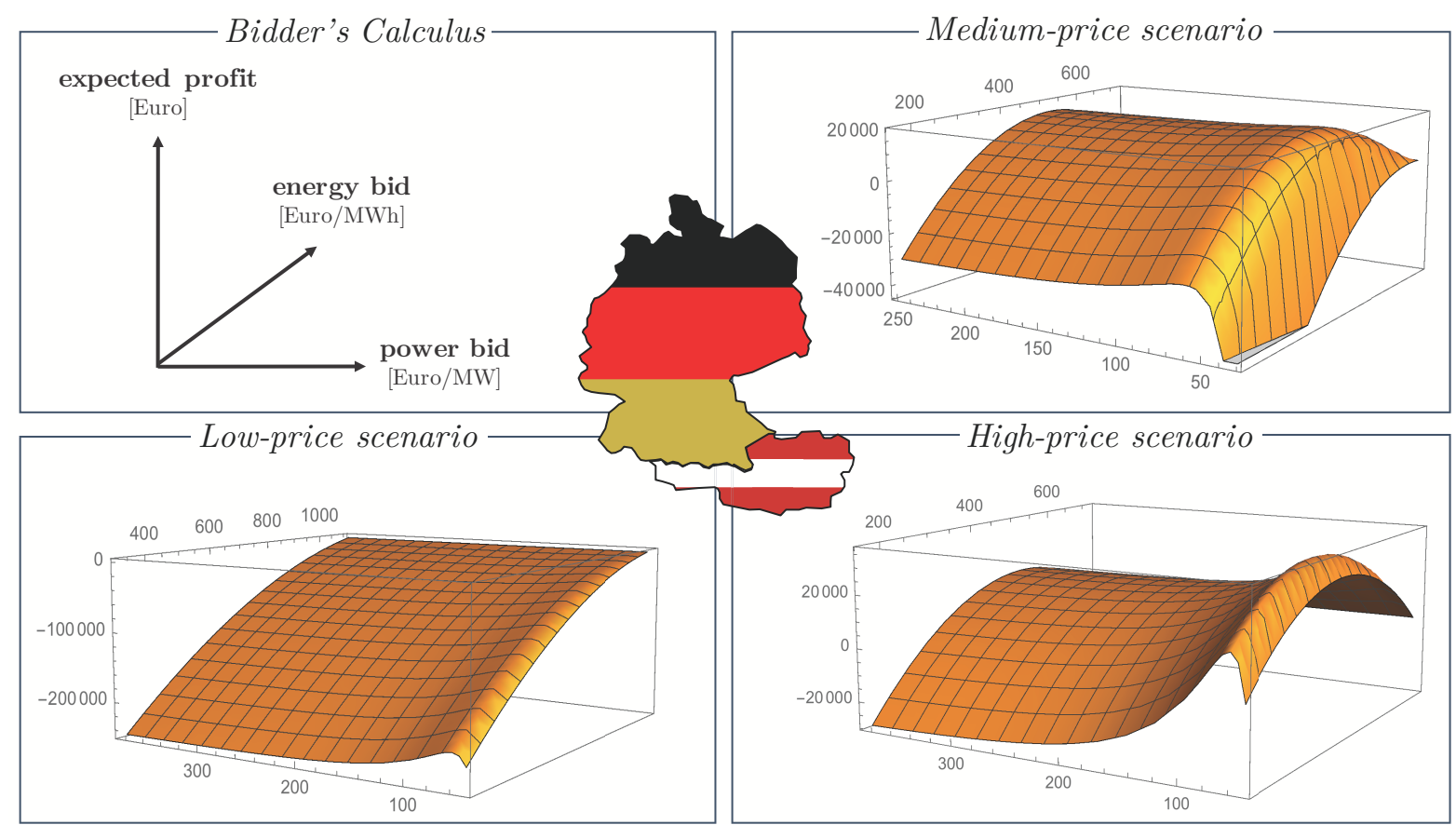

Figure 1: Graphical representation of the bidder's calculus for different price scenarios in Austrian and German balancing power auctions. 


\section{NOMENCLATURE}

$\begin{array}{ll}\text { Abbreviations } & \\ \text { aFRR } & \text { automatically-activated Frequency Restoration Reserve } \\ \text { mFRR } & \text { manually-activated Frequency Restoration Reserve } \\ \text { Euro/MW } & \text { Euro per Megawatt } \\ \text { Euro/MWh } & \text { Euro per Megawatt hour } \\ \text { FCR } & \text { Frequency Containment Reserve } \\ \text { HT } & \text { Haupttarif (main period) } \\ \text { MW } & \text { Megawatt } \\ \text { MWh } & \text { Megawatt hour } \\ \text { NT } & \text { Nebentarif (sub-period) } \\ \text { TSO } & \text { Transmission System Operator }\end{array}$




\section{Mathematical Notation}

\begin{tabular}{|c|c|}
\hline$\pi$ & supplier's profit \\
\hline$\pi_{P}$ & supplier's profit of the power bid \\
\hline$\pi_{E}^{e}$ & supplier's expected profit of the energy bid \\
\hline$b$ & power bid \\
\hline$b^{*}$ & optimal power bid \\
\hline$b_{\min }$ & lowest awarded power bid \\
\hline$b_{\max }$ & highest awarded power bid \\
\hline$c$ & capacity costs \\
\hline$d$ & reserving period \\
\hline$k$ & calling costs \\
\hline$n$ & demand probability \\
\hline$p$ & energy bid \\
\hline$p^{*}$ & optimal energy bid \\
\hline$p_{\min }$ & lowest energy bid \\
\hline$p_{\max }$ & highest energy bid \\
\hline$q$ & power offer \\
\hline$r_{R U}$ & price for ramping up the power station \\
\hline$r_{U S}$ & price for using the power station \\
\hline$x$ & position in the merit-order of energy bids \\
\hline$D$ & balancing power demand \\
\hline$G$ & supplier's (subjective) acceptance probability \\
\hline$P_{\text {min }}$ & minimal load \\
\hline
\end{tabular}




\section{INTRODUCTION}

In an electric energy (henceforth energy) system with a high share of volatile renewable energy sources, ancillary services for the grid become increasingly important. In general, three types of ancillary services, which support the supply reliability in distinct cases, can be distinguished. ${ }^{1}$ In the first case, the market clearing on the wholesale market fails because energy demand is higher than energy supply. ${ }^{2}$ Then, capacity mechanisms ensure the supply reliability by activating standby plants (Cramton \& Ockenfels, 2012). In the second case, the market clearing on the wholesale market was successful, but grid restrictions do not allow a certain allocation, e.g., because of internal gird bottlenecks. ${ }^{3}$ In this case, the allocation on the wholesale market must be changed (by a so-called "redispatch"), i.e., contracted energy producers who intend to transmit energy via the bottleneck must lower their energy supply. The missing energy supply is replaced by suppliers that are spatially behind the bottleneck. If the market clearing on the wholesale market was successful and the allocation is aligned with the grid restrictions, a third case may arise, and this is the case which is analyzed in this paper: the predicted energy demand and supply do not match (e.g., because of a sudden overproduction of a photovoltaic plant). Then, the energy demand and supply must be balanced instantaneously to stabilize the frequency in alternating current grids. This short-term operability of the grid is ensured with the help of balancing power.

For integrating balancing power into the energy system, there exist two implementation options with respect to the minimization of the expected energy costs: firstly, integrated or coupled co-optimization (usually applied in North America) and, secondly, decoupled co-optimization (usually applied in Europe). Co-optimization means that the allocations on the wholesale market and on the balancing power market are optimized simultaneously. Decoupled co-optimization means that the allocations on the two markets are determined separately (Ellison, Tesfatsion, Loose, \& Byrne, 2012).

\footnotetext{
${ }^{1}$ The actual implementation and classification of ancillary services depends on the specific country. For Germany, see for example Schweizer and Mattis (2016).

${ }^{2} \mathrm{~A}$ reason for this may be that the continuous penetration of renewable energy sources leads to a decline in wholesale market prices, which threatens the profitability of conventional power plants.

${ }^{3}$ In Germany, the energy production of wind power plants in the Northern Sea often overextends the grid capacities to Southern Germany, requiring a redispatch of energy production (Ocker \& Ehrhart, 2017b).
} 
In the Austrian and German energy systems, the transmission system operators (TSOs) are legally obliged to organize balancing power in an open market at the lowest possible cost. Therefore, they conduct auctions for the procurement of balancing power for both the Austrian and the German control area (regelleistung.net, 2018; Austrian Power Grid, 2018). Here, prequalified suppliers offer balancing power in three different markets: Frequency Control Reserve (FCR), automatically-activated Frequency Restoration Reserve (aFRR), and manually-activated Frequency Restoration Reserve (mFRR) (ENTSO-E, 2017).

These three balancing markets are distinguished by the reaction time of balancing power being available to the grid. In Austria and Germany, FCR needs to be available 30 seconds, aFRR five minutes, and mFRR 15 minutes after an imbalance causing event. Since this is either caused by underproduction (e.g., emergency stop of a plant) or overproduction (e.g., massive production by a wind park), there are two different types of balancing power: positive and negative balancing power. The first is needed if not enough energy is supplied, and the latter if too much energy is produced. A power plant that provides positive balancing power increases its load level, while a power plant that supplies negative balancing power decreases its load level. In most European countries, the aFRR market has by far the highest balancing power demand and, thus, is the most important short-term ancillary service. For this reason, this paper focuses on the Austrian and German aFRR markets, which share the same market design. The Austrian and German aFRR markets can further be separated with respect to the time period for which energy needs to be made available. There are two different reserving periods, the main period (Haupttarif, HT) from Monday to Friday from 8am to 8pm (60 hours per week), and the sub-period (Nebentarif, NT) that covers the rest of the time (108 hours per week). Hence, four separate aFRR auctions are conducted weekly both in Austria and in Germany (regelleistung.net, 2018; Austrian Power Grid, 2018).

We present a decision-theoretic approach for deriving bidding strategies of suppliers in the Austrian and German aFRR auctions. By comparing our findings with empirical data, we find accordance with theoretic predictions for suppliers' market behavior. Thus, this paper contributes to the discussion of balancing power market design. This is of relevance because the European Commission intends to harmonize the European balancing power auctions in the near future, and the current proposal of a common design is strongly geared to the 
Austrian and German auctions (European Commission, 2017).

The remainder of this paper is structured as follows. The first section refers to related literature. The second section considers the aFRR market environments in Austria and Germany. The third section presents a bidder's decision-theoretic calculus. In the fourth section, a numerical case study of the bidder's calculus in different market scenarios is illustrated. The fifth section confronts the theoretic predictions with empirical market data from Austria and Germany. The last section concludes and illustrates further need of research.

\section{Related Literature}

This literature review considers mainly research on multi-unit auctions, since most balancing power markets worldwide use auctions as market mechanisms. In a balancing power auction, the auctioneer (e.g., the regulator, TSO or Independent System Operator) demands multiple goods (i.e., multiple units of reserved power). ${ }^{4}$ In multi-unit auctions, the bidders' strategy spaces are substantially larger and more diverse than in single-item auctions, and often a theoretic solution in form of a bidding equilibrium does not exist. ${ }^{5}$ Nevertheless, multiple analyses help to develop a better understanding of information acquisition (Gretschko, Rasch, \& Wambach, 2014), information disclosure (Bergemann \& Wambach, 2015), collusion (Skrzypacz \& Hopenhayn, 2004; Hortacsu \& Puller, 2008) or competing sellers (McAfee, 1993). Ocker, Ehrhart, and Belica (2018) show that there is a unique symmetric equilibrium in the Austrian and German aFRR auctions. Yet, empirical market data deviates substantially from the theoretic findings, in particular, the submitted bids are on a far higher price level. Ocker and Ehrhart (2017a) argue that suppliers were able to establish these high price levels by adjusting their bids to a "focal point," i.e., the highest accepted bid of the previous auction results. Motivated by this finding, we present a bidder's decision-theoretic calculus that allows the integration of previous auction results as future price expectations and compare the results with empirical market data from Austria and Germany.

Furthermore, we refer to scoring auctions. These are multi-attributive auctions, in which,

\footnotetext{
${ }^{4}$ For examples of multi-unit auctions see Ausubel, Cramton, Pycia, Rostek, and Weretka (2014).

${ }^{5}$ For an overview see Ausubel et al. (2014) and for the relevance in energy markets see Wolfram (1997).
} 
beside the price, also other attributes are considered for the assessment of bids, e.g., price, quality, or time of delivery (Che, 1993; Branco, 1997; Asker \& Cantillon, 2008, 2010). For example, for the construction of highway roads, both the time and the costs are of importance (Herbsman, Chen, \& Epstein, 1995). Consequently, all parameters that are of relevance for winner selection need to be comprised in a so-called "scoring rule" (Bichler \& Kalagnanam, 2005; David, Azoulay-Schwartz, \& Kraus, 2006). Regarding balancing power markets, Bushnell and Oren (1995) and Chao and Wilson (2002) theoretically examine different scoring auctions. They describe essential elements for scoring rules to ensure an efficient allocation. As shown in Ocker et al. (2018), the Austrian and German aFRR auctions, with the two attributes power and energy, theoretically ensure an efficient market outcome. Nevertheless, empirical auction results point at a more rigorous investigation regarding the interplay of these two bid elements since they are not in line with the theoretic predictions. Therefore, we provide a detailed theoretic analysis of the two attributes' interconnection, and complement the findings by several numerical examples.

The literature on balancing power auctions contains several approaches. Wen and David (2002) present a stochastic optimization model for the derivation of the bidding strategies. Kamat and Oren (2002) discuss efficiency properties of different auction formats for balancing power in the USA. Müller and Rammerstorfer (2008) categorize different elements suitable for the design of balancing power markets. Just and Weber (2008) illustrate the interplay between balancing power markets and spot energy markets. Rammerstorfer and Wagner (2009) provide and empirical assessment of the effects associated with an reorganization of the German balancing power market. Holttinen (2012) examines experiences and future challenges of wind power integration and relates it to balancing power. Heim and Götz (2013) find evidence that suppliers strategically withheld capacities in the German balancing power auctions, leading to inefficient outcomes. Bessa, Moreira, Silva, and Matos (2014) discuss how renewable energy variability and uncertainty in power systems operation can be handled, e.g., how system operators manage their systems based on forecasts of renewable generation. Müsgens, Ockenfels, and Peek (2014) discuss the economic fundamentals that govern market design and behavior in the German markets. Hirth and Ziegenhagen (2015) connect renewable energy sources to balancing power markets and demand for a "more rig- 
orous evaluation of the price development." Söder (2016) analyses balancing challenges in sustainable and smart energy systems with $100 \%$ renewable power supply. Empirical analyses reveal a large heterogeneity among the European auction designs for balancing power markets, indicating that there is no predominant market design (Ocker, Braun, \& Will, 2016; Ocker, 2017). Ocker et al. (2018) provide a game-theoretic model for the current Austrian and German aFRR auction as well as for the proposed European-wide aFRR auction. Furthermore, Ocker and Ehrhart (2017a) present empirical evidence for collusive behavior in the German balancing power markets, i.e., suppliers coordinate on non-competitive price levels. We relate to the latest literature and present an investigation of the Austrian and German aFRR auctions outcomes in the time span from January 2014 to May 2016. For this, we compare the theoretic findings with empirical bids to verify whether the identified bidding strategies are applied by the suppliers.

\section{Austrian and German aFRR Market Environment}

In the current Austrian and German aFRR market design, a bid consists of three components: A power offer, a power bid and an energy bid. ${ }^{6}$ The power offer encompasses the amount of balancing power offered [Megawatt, MW], the power bid [Euro/MW] compensates for keeping power available for the aFRR market, and the energy bid [Euro/MWh] compensates for the actual delivery of balancing energy. The scoring rule, which determines the winners of the auction, is based on the power bid exclusively, and neglects the value of the energy bid. Therefore, we structure our analysis along the power and energy bid: Step one for the acceptance of the power bid, and step two for being called with the energy bid.

Awarded power bids $b$ with the corresponding power offers $q$ are remunerated by applying the pay-as-bid rule, i.e., an awarded supplier's compensation equals her bid. ${ }^{7}$ The power bids are first arranged in increasing order and then, starting with the lowest power bid, awarded till the balancing power demand $D$ is met. Hereby, $b_{\min }$ and $b_{\max }$ denote the lowest and the highest awarded power bid. The last awarded power bid is only accepted partially if not all

\footnotetext{
${ }^{6}$ Note that power offer and power bid are also referred to as capacity offer and capacity bid.

${ }^{7}$ For a discussion of uniform pricing see Ocker et al. (2018).
} 
of the power offer is needed to satisfy the demand. This special case will not be part of our analysis. ${ }^{8}$ The rule of awarding a power bid is illustrated in Figure 2 for a supplier $j$. Here, the power offer $q_{j}$ with the respective power bid $b_{j}$ is accepted. Furthermore, a power bid that is not awarded is depicted: The one that has a power bid greater than $b_{\max }$.

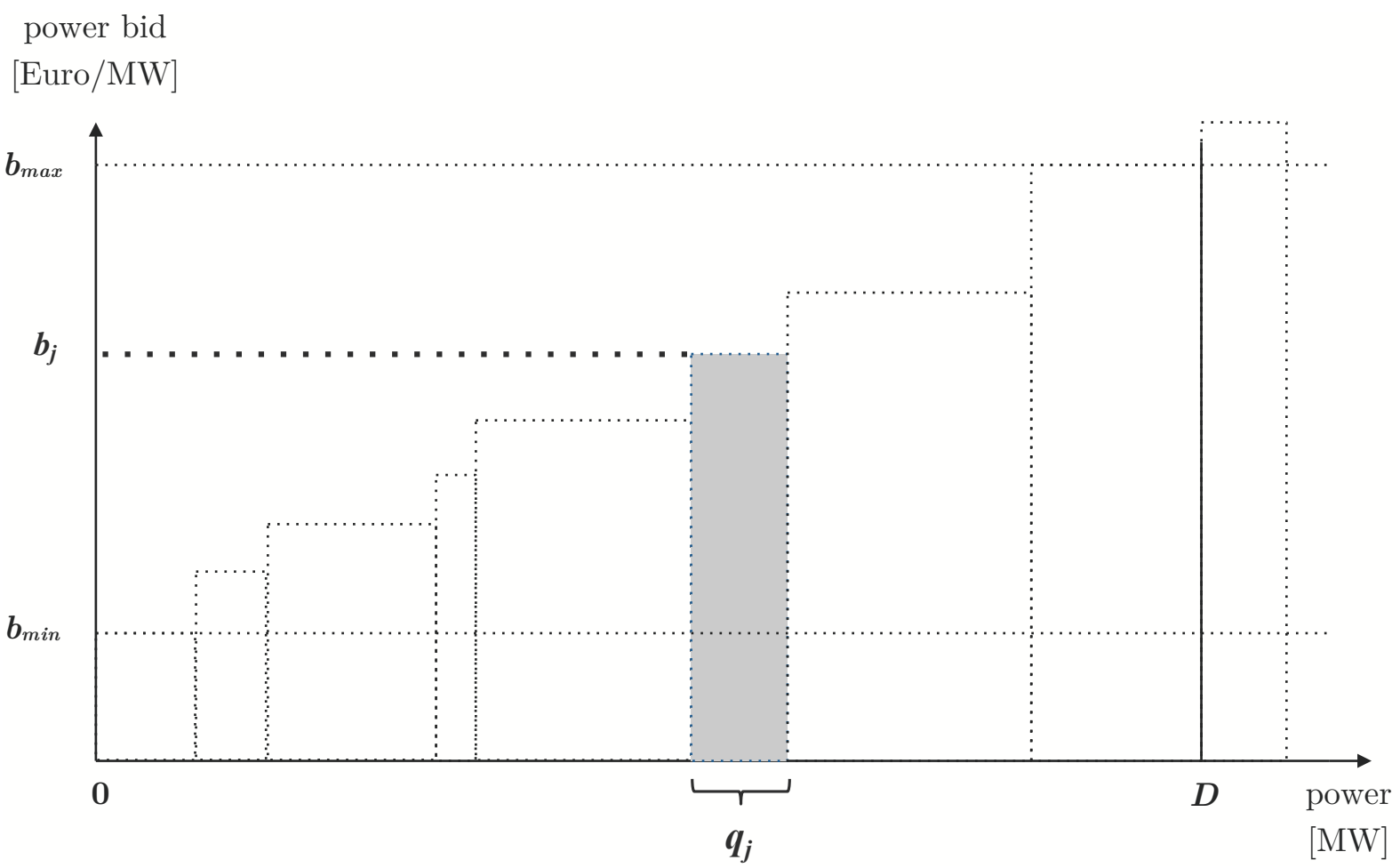

Figure 2: Step one of the operating principle in the Austrian and German aFRR auctions: Winner determination (positive and negative aFRR).

With an increasing power bid, the probability for being awarded ("acceptance probability") decreases. A supplier's (subjective) acceptance probability, which is denoted by $G(b)$, captures the supplier's beliefs about his opponents' power bids. The profit of the power bid is the margin between a supplier's power bid and costs for keeping power available, the so-called "capacity costs" (see Section Capacity Costs), multiplied with the power offer. For example, with a power bid of $b=200$ Euro/MW, capacity costs of $c=100$ Euro/MW and a power offer $q=10 \mathrm{MW}$, the profit of the power bid is given by:

$$
(200-100) \text { Euro/MW } \cdot 10 \mathrm{MW}=1,000 \text { Euro. }
$$

\footnotetext{
${ }^{8}$ For a discussion of this special case see Swider and Weber (2007).
} 
If the power bid is accepted, suppliers can generate profits with the energy bid $p$ as well. That is the case if the provided power $q$ is actually needed for stabilizing the grid frequency. In the auction, the energy bids of suppliers with awarded power bids are arranged in increasing (decreasing) order in the positive (negative) market, i.e., the lowest (highest) energy bid will be utilized most in the positive (negative) market. The reason for this distinction among the positive and negative market is due to the official nomenclature of the TSOs: In the positive market, the lowest position in the merit-order is assigned to the lowest submitted energy bid, whereas in the negative market, the lowest position in the merit-order is assigned to the highest submitted energy bid. Consequently, a negative energy bid in the negative market corresponds to a positive energy bid in the positive market (i.e., the TSOs pay the supplier for the delivery of balancing energy), and a positive energy bid in the negative market states the willingness of a supplier to pay money to the TSOs for being called. ${ }^{9}$ The applied activation strategy is a merit-order of energy bids. The profit of the energy bid is thereby influenced by the position in the merit-order. This is depicted in Figure 3 for the positive market for a supplier $j$. Here, the energy bid $p$ expresses the price a supplier asks for delivering one unit of positive balancing energy.

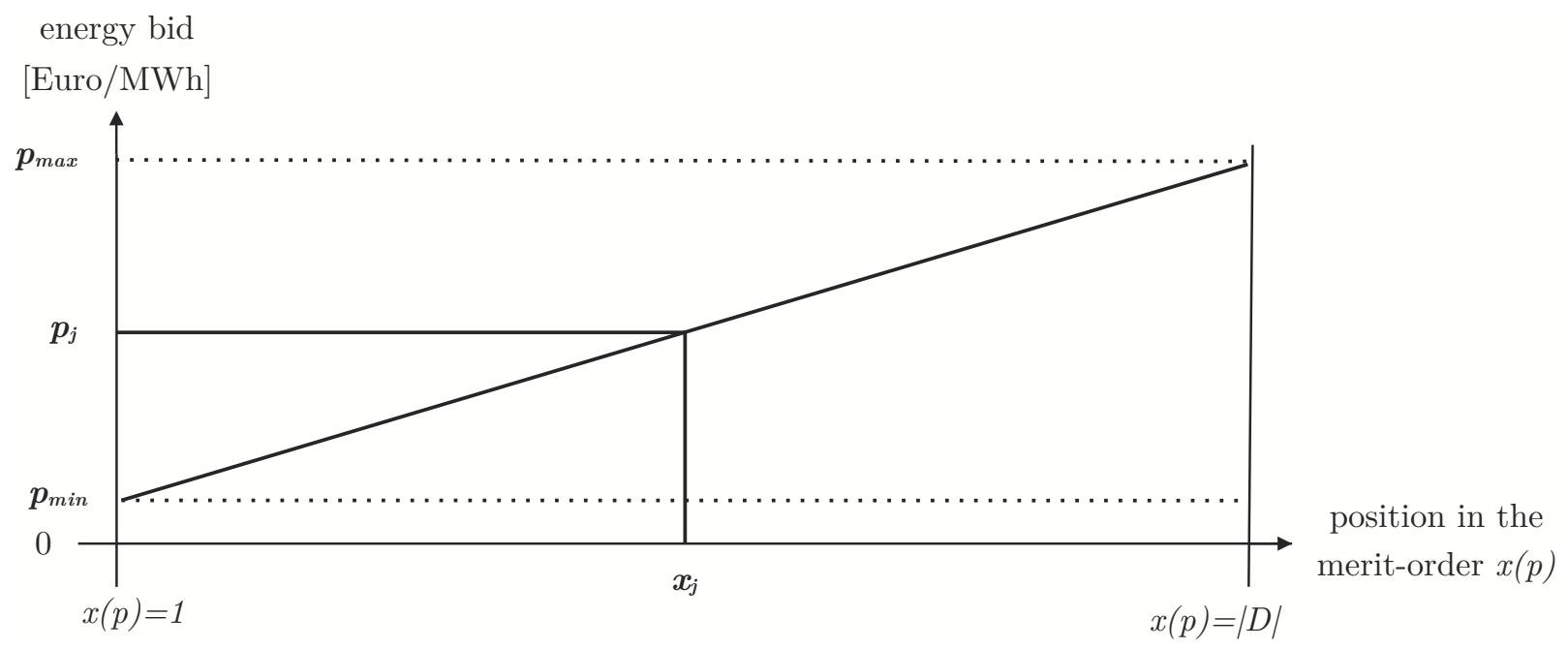

Figure 3: Step two of the operating principle in the Austrian and German aFRR auctions: Position in the merit-order of the energy bids (positive aFRR).

\footnotetext{
${ }^{9}$ Technically, suppliers can also submit a negative energy bid in the positive market. However, this is not sensible from an economical perspective.
} 
The relative height of an energy bid $p$ determines its position(s) $x(p)$ in the merit-order of the energy bids. ${ }^{10}$ The lower the energy bid, the lower are its positions in the merit-order, i.e., $x(p)$ is increasing in $p$. The lowest energy bid $p_{\min }$ is at the first position and the highest energy bid $p_{\max }$ at the last position of the merit-order.

In the negative aFRR market, suppliers are willing to pay money to the TSOs in order to be positioned at the very beginning of the merit-order. Recall that in the negative market, a supplier provides balancing energy by, typically, decreasing the load level of her power plant since too much energy is supplied to the power system. Furthermore, energy delivery is still compensated on short-term or long-term markets. Hence, suppliers can save their variable costs by bidding a positive energy bid up to the height of the variable costs. In Figure 4, the merit-order of the energy bids for the negative market is illustrated. Here, a supplier $j$ is willing to pay money to the TSOs for being utilized for the delivery of balancing energy.

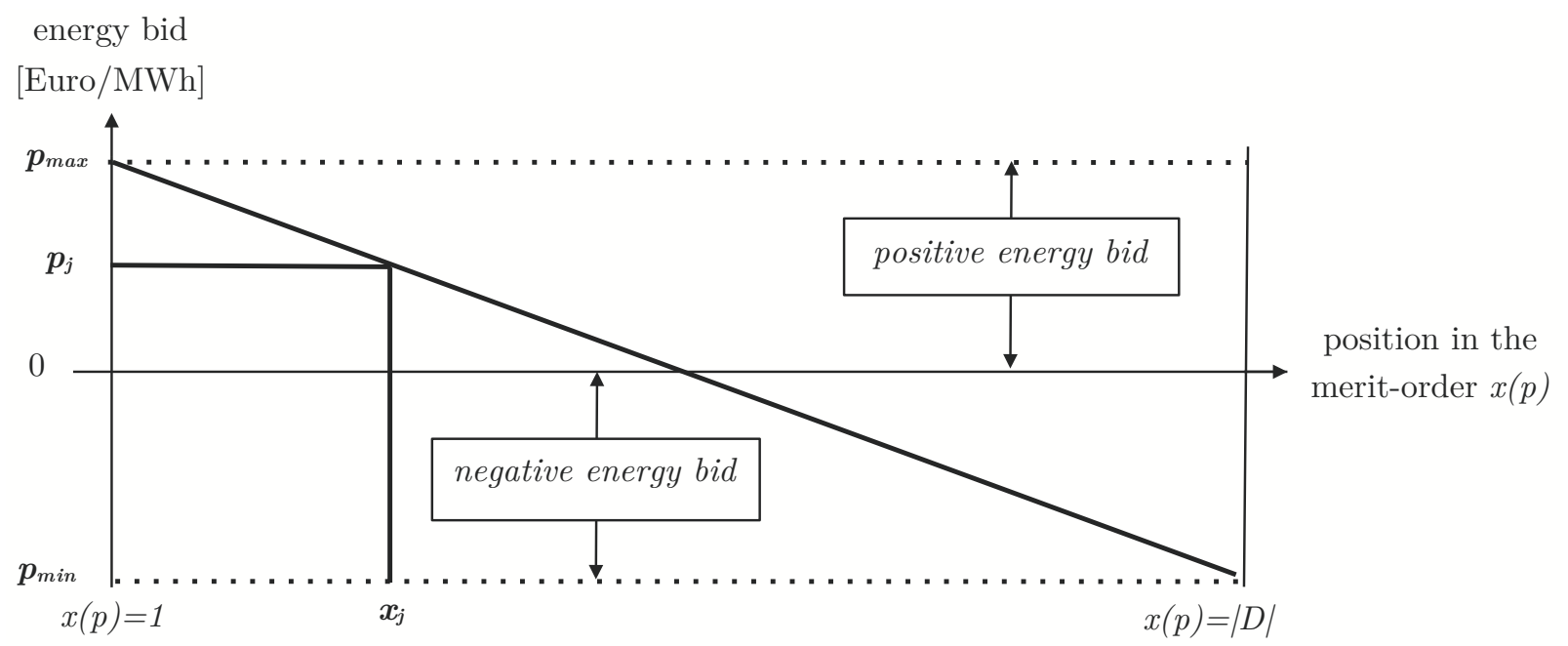

Figure 4: Step two of the operating principle in the Austrian and German aFRR auctions: Position in the merit-order of the energy bids (negative aFRR).

Each possible position in the merit order comes with a probability of being called. This is stated in the so-called "demand probability" $n(x(p))$, which is illustrated in Figure 5. Note, the demand probability reflects the suppliers subjective (ex-ante) beliefs about the demand frequency for the respective reserving period and is strictly (but not necessarily linearly) decreasing in $x(p)$ in both the positive and negative market. The demand probability $n(x(p))$

\footnotetext{
${ }^{10}$ Each MW of an awarded power bid occupies one position.
} 
decreases with the position $x(p)$. Hence, the highest demand probability is assigned to the lowest (highest) energy bid in the positive (negative) market.

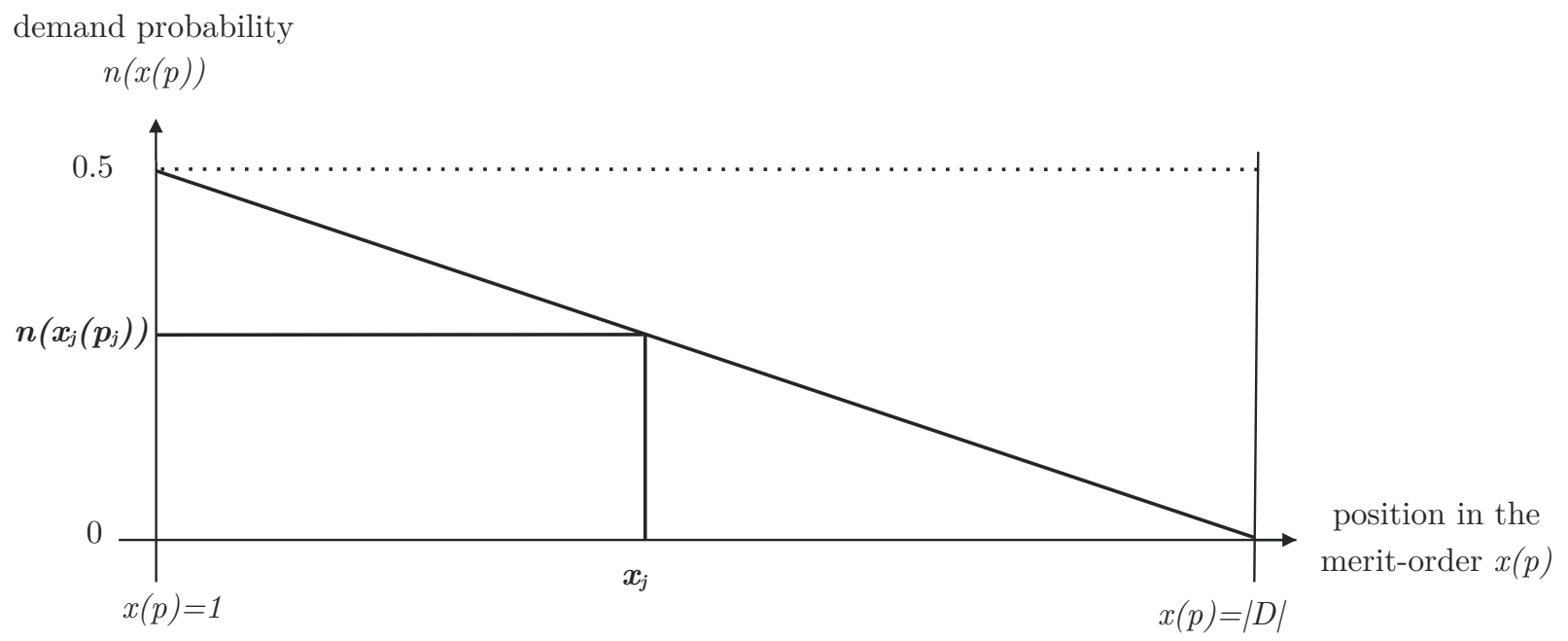

Figure 5: Step two of the operating principle in the Austrian and German aFRR auctions: Demand probability (positive and negative aFRR).

In line with empirical data, we assign for both the positive and negative aFRR market the highest probability of being called to $50 \%$, i.e., the demand probability of positive and negative aFRR is the same. To demonstrate how the profit of the energy bid is calculated, we present an example for the positive aFRR in the main period. For that, we set the energy bid to a value of $p=100$ Euro/MWh resulting in position $x(p)=500$ in the merit-order. Let the variable costs of the power plant be 50 Euro/MWh and the power offer $q=10 \mathrm{MW}$. For the position 500, we set the demand probability to 0.38 , i.e., in $38 \%$ of the reserving period with $d=60$ hours (resulting in $22.8 \mathrm{~h}$ ) balancing energy is actually called. Hence, the profit of the energy bid is given by:

$$
(100-50) \text { Euro/MWh } \cdot 10 \mathrm{MW} \cdot 22.8 \mathrm{~h}=11,400 \text { Euro. }
$$




\section{Bidder's Decision-theoretic Calculus for the Austrian and German aFRR Auctions}

In the following, a bidder's decision-theoretic calculus for the aFRR auctions is presented. First, we examine the different components of the bidder's calculus. Then, conditions for optimal power and energy bids are presented that allow the integration of the suppliers' price expectations. Finally, the cost structures and stochastic influences are discussed.

\section{Components of the Bidder's Calculus}

The bidder's calculus in this section applies to the positive and negative aFRR markets. ${ }^{11}$

If a supplier's power bid is awarded, his profit $\pi(b, p)$ consists of two elements, the profit of the power bid $\pi_{P}(b)$ and the expected profit of the energy bid $\pi_{E}^{e}(p)$ :

$$
\pi(b, p)=\left\{\begin{aligned}
\pi_{P}(b)+\pi_{E}^{e}(p), & \text { if } b \text { is awarded } \\
0, & \text { else. }
\end{aligned}\right.
$$

The profit of the power bid is given by

$$
\pi_{P}(b)=b q-c q=(b-c) q
$$

where $c$ denotes the capacity costs per MW (see Section Capacity Costs).

A supplier is assumed to maximize her expected profit

$$
\pi^{e}(b, p)=G(b)\left(\pi_{P}(b)+\pi_{E}^{e}(p)\right)
$$

where $\pi_{E}^{e}(p)$ can be expressed by the calling costs $k$ (see Section Calling Costs), the position in the merit-order $x(p)$, the demand probability $n(x(p))$, and the reserve period $d$ :

$$
\pi_{E}^{e}(p)=(p q-k q) d \cdot n(x(p))=(p-k) q d \cdot n(x(p))
$$

With (4) and (6), the expected profit can be written as

$$
\pi^{e}(b, p)=G(b) \cdot q \cdot((b-c)+(p-k) d \cdot n(x(p))) .
$$

\footnotetext{
${ }^{11}$ For our approach to derive optimal bidding strategies we adjust the profit function of Bushnell and Oren (1995) to the Austrian-German aFRR auction design.
} 


\section{Optimal Power and Energy Bid}

For maximizing the expected profit in (7), we compute the first-order conditions by differentiating the expected profit $\pi^{e}(b, p)$ with respect to the two bid components $b$ and $p .{ }^{12}$ The first-order condition for the optimal power bid $b^{*}$ leads to the following condition:

$$
b^{*}=c-\left(p^{*}-k\right) d \cdot n\left(x\left(p^{*}\right)\right)-\frac{G\left(b^{*}\right)}{G^{\prime}\left(b^{*}\right)} .
$$

$G^{\prime}(b)$ denotes the derivative of $G(b)$ with $G^{\prime}(b)<0$, i.e., the higher the bid, the lower is the acceptance probability. According to (8), the capacity costs are the basis for calculating the optimal power bid. The term $\left(p^{*}-k\right) d \cdot n\left(x\left(p^{*}\right)\right)$, which reflects the expected profit of the energy bid per MW, is subtracted from $c$. That is, the expected profit of the energy bid is taken into account for calculating the optimal power bid. The higher the expected profit of the energy bid, the lower is the optimal power bid. The term $\frac{G\left(b^{*}\right)}{G^{\prime}\left(b^{*}\right)}$ is negative because the acceptance probability is positive and its derivative is negative. Thus, the absolute value of this term is added to $c$. This markup is due to the pay-as-bid rule and corresponds to the markdown in sales auctions that is called "bid-shading" (Milgrom \& Weber, 1982; Kremer \& Nyborg, 2009; Ausubel et al., 2014). The amount of the markup depends on the supplier's beliefs about the competition level (Myerson, 1981; Kahn, Cramton, Porter, \& Tabors, 2001). The higher, ceteris paribus, the acceptance probability, the higher is the markup. Note that the optimal power bid is independent of the power offer $q$.

The first-order condition for the optimal energy bid $p^{*}$ leads to the following condition:

$$
p^{*}=k-\frac{n\left(x\left(p^{*}\right)\right)}{n^{\prime}\left(x\left(p^{*}\right)\right) \cdot x^{\prime}\left(p^{*}\right)} .
$$

Note that the fraction is always negative in the positive market: The derivative $x^{\prime}\left(p^{*}\right)$ is positive (the higher the energy bid, the higher is the position in the merit-order), while the derivative $n^{\prime}\left(x\left(p^{*}\right)\right)$ is negative (the higher the position, the lower is the demand probability). In the negative market, the fraction is always positive: The derivative $x^{\prime}\left(p^{*}\right)$ is negative (the higher the energy bid, the lower is the position in the merit-order), while the derivative $n^{\prime}\left(x\left(p^{*}\right)\right)$ is also negative (the higher the position, the lower is the demand probability). According to (9), the optimal energy bid is independent of the power offer $q$ as well as of

\footnotetext{
${ }^{12}$ For our assumptions on $G(b), x(p)$, and $n(x(p))$ the second-order conditions are fulfilled.
} 
the optimal power bid $b^{*}$. The latter can be interpreted as follows: Since the acceptance of the power bid is a necessary condition for generating further profits with the energy bid, the power bid needs not to be taken into account for the optimal energy bid. Again, the cost element, here the calling costs $k$, form the basis for calculating $p^{*}$. Since the subtracted term in (9) is negative (positive) in the positive (negative) market, it expresses a markup (markdown). ${ }^{13}$ This is again due to the pay-as-bid rule and also corresponds to bid-shading in sales auctions. That is, a supplier does not bid her true calling costs (Ocker et al., 2018).

\section{Operation Modes of Power Plants}

To quantify the capacity costs (see Section Capacity Costs) for providing aFRR, a supplier has to take into account the operation mode of her power plant. Capacity costs equal opportunity costs if the power station is already running and offering energy at the spot market (spinning reserve) (Müsgens et al., 2014). If, however, a power station is only running for providing aFRR, capacity costs cover all resulting costs from running the power plant and need to be covered by the earnings of participating on the aFRR market.

This raises a simple but crucial question, that a supplier has to answer before calculating her power bid: How will the power plant run, if it is not used for providing aFRR? With this approach, capacity costs that result from the supply of aFRR can be calculated correctly. We follow the nomenclature of Müsgens et al. (2014), who call a power plant inframarginal (extramarginal), if the variable costs of a power plant are smaller (greater) than the relevant market price (i.e., the price determined by short- or long-term contracts). Three different operation modes are linked to this classification.

Operation Mode 1 captures power plants that are inframarginal, i.e., spinning reserve. These power plants generate profits by selling power, e.g., at the spot market. Thus, the power plant is running independently of the decision whether to provide aFRR or not. In the case that the variable costs are greater than the relevant market price, Operation Mode 2 applies. If such a power plant participated at the spot market, it would generate losses

\footnotetext{
${ }^{13}$ In the positive market, for example, a supplier with $k=50$ Euro/MWh always bids higher than her calling costs. In the negative market, the same supplier is willing to pay up to 50 Euro/MWh to the TSOs for being called (i.e., submitting a positive energy bid), however, always bids lower than her calling costs.
} 
and therefore would not sell energy at the spot market. Since the relevant market price can be flexible within minutes, there is room for a third mode of operation. This is the case if a power plant is inframarginal at one point and extramarginal at another point during the period of providing balancing power. In this case, capacity costs can in parts also be covered by the spot market. This is especially relevant for power plants that have variable costs close to the market price.

\section{Capacity Costs}

The capacity costs $c$ in Euro/MW consist of all costs that occur for holding power in the reserve period (Hirth \& Ziegenhagen, 2015). Depending on whether a power plant is infraor extramarginal, different cost components need to be taken into account.

The capacity costs in the positive aFRR market for an inframarginal power plant consist of the opportunity costs which equal the lost profits of not trading power at an alternative power market (Müsgens et al., 2014). Hence, the opportunity costs are the margin between the relevant market price (e.g., the wholesale market price) and the variable costs multiplied with the reserve period $d$. Since the market price is volatile and unknown at the time of the auction, suppliers necessarily have to base their decisions on expectations about this price.

If a power plant is extramarginal, it works on minimal load $P_{\min }$ in order to keep losses as low as possible. These losses, which occur because the variable costs are greater than the relevant market price, are assigned to the capacity costs. Furthermore, ramp-up costs and usage costs play a role for extramarginal power stations. It is assumed that a power plant has a price for ramping up $r_{R U}$ as well as a price for using the power station $r_{U S}$ for the time to hold aFRR available. ${ }^{14}$ These two cost components are assigned to the capacity costs.

In the negative aFRR market, the capacity costs are slightly different. Since all of the energy of an inframarginal power plant can be sold with a profit, opportunity costs and, therefore, capacity costs, are zero (Hirth \& Ziegenhagen, 2015). If the power plant

\footnotetext{
${ }^{14}$ Depending on what operation mode is applied, the power plant could ramp up and ramp down more than once. These costs are summed up in $r_{R U}$. The same applies for the usage costs. Usage costs are measured in Euro per hour of running the power plant and need to be multiplied by the time the power plant provides aFRR. Total usage costs are given by $r_{U S}$.
} 
is extramarginal, the power plant has to run at the load level $P_{\min }+q$ in order to be able to reduce power if called (Müsgens et al., 2014). Hence, extramarginal power plants generate losses that depend on the difference between variable costs and the market price. This difference multiplied with the product of the load level $P_{\min }+q$ and the aFRR time determines the total loss per MW. Ramp-up and usage costs occur for extramarginal power plants only.

\section{Calling Costs}

The costs $k$ in Euro/MWh for delivering energy influence the energy bid. These costs occur if balancing energy is actually called by the TSOs, which is executed automatically according to the merit-order. For the positive aFRR market, these are the variable costs of the power plant. In the negative aFRR market, these costs are actual savings for both inframarginal and extramarginal power plants. The reason for this is that suppliers are still compensated with the relevant market price but save the variable costs of running the power plant. The minimum willingness to accept is determined by the variable costs of the power plant.

\section{Empirical Identification of Acceptance and Demand Probability}

In the following, we exemplarily consider the German positive market in the main period because this is the easiest case to compute (Müsgens et al., 2014).

To generate optimal power and energy bids, a supplier's beliefs about the competition level and, thus, the power and energy price levels for the aFRR auction have to be taken into account. These beliefs are described by the subjective acceptance probability $G(b)$ and demand probability $n(x(p))$ (see Section Austrian and German aFRR Market Environment). Remember, $G(b)$ is decreasing in the power bid $b$ and $n(x(p))$ is decreasing in the merit-order position $x(p)$. What needs to be specified are the shapes and the intervals on which these functions are defined. The interval will later be used to describe different market scenarios: For a low-price (high-price) period, the distributions for the expected power and energy bids are defined on lower (higher) values.

Supported by Ocker and Ehrhart (2017a), we assume that suppliers do not reveal their 
actual costs in their bids, but take into account previous auctions results when deriving their beliefs about the power price level in the next auction (modeled by the acceptance probability $G(b)) .{ }^{15}$ For defining different price levels of the power bid, we consider empirical German aFRR market data from January 2012 until December 2013 (105 auctions). In Germany, the TSOs publish the lowest and highest awarded power bids as well as the mean weighted power bid of every auction (regelleistung.net, 2018). For describing different price levels, we consider the highest power bids awarded $b_{\text {max }}$, which is shown in Figure 6.

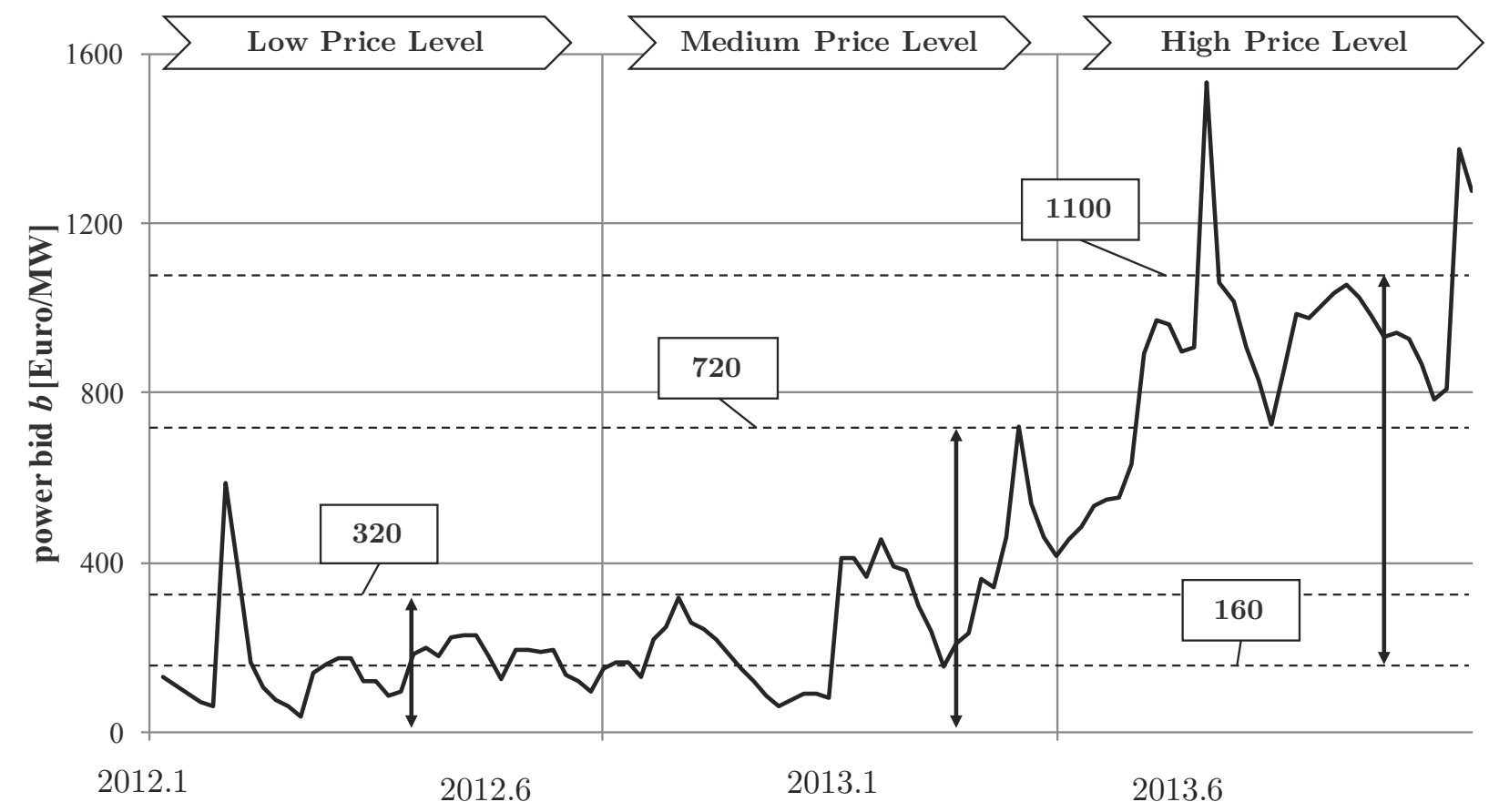

Figure 6: Development of the highest awarded power bid $b_{\max }$ from January 2012 to December 2013 with a total of 105 auctions (regelleistung.net, 2017).

We consider it as suitable for our analysis to divide the total range of $b_{\max }$ into three intervals. ${ }^{16}$ Within the first weeks, $b_{\max }$ moved in an interval of about $[0,320]$ Euro/MW. After that, there was a rise in prices leading to a maximum $b_{\max }$ of about 720 Euro/MW.

\footnotetext{
${ }^{15}$ Theoretic and empirical evidence for similar behavior of suppliers due to the frequent repetition of an auction in other markets can be found in Macatangay (2002); Cramton and Schwartz (2000); Ehrhart (2001); Lu, Gupta, Ketter, and Heck (2014); Ishii (2014).

${ }^{16}$ The identification of price intervals serves only as input for our numerical example in the following section, illustrating the basic principle of bidding strategies. Hence, a more elaborate method for defining the price intervals (e.g., for both the Austrian and German aFRR auctions) is not necessary.
} 
Hence, a second interval can be defined with boundaries of approximately [0, 720] Euro/MW. Ultimately, prices increased further, leading to boundaries for an interval of about $[160,1,100]$ Euro/MW (neglecting the outliers at week 84 and 104).

Note that the demand for positive aFRR was approximately on the same level during the considered time period from January 2012 until December 2013 (Ocker \& Ehrhart, 2017a). Therefore, we assume that suppliers derive their subjective acceptance probability from the corresponding power price interval. For simplicity, we assume a linear shape of the acceptance probability, leading to the functions presented in the first part of Table $1 .{ }^{17}$

\begin{tabular}{lcc} 
Power bid price level & Interval & $G(b)$ \\
\hline Low price level & {$[0,320]$} & $1-\frac{1}{320} \cdot b$ \\
Medium price level & {$[0,720]$} & $1-\frac{1}{720} \cdot b$ \\
High price level & {$[160,1,100]$} & $\frac{55}{47}-\frac{1}{940} \cdot b$ \\
\hline Energy bid price level & Interval & $x(p)$ \\
\hline Low price level & {$[40,250]$} & $-380+\frac{1,990}{210} \cdot p$ \\
Medium price level & {$[70,1,120]$} & $-132+\frac{1,990}{1,050} \cdot p$ \\
High price level & {$[90,6,000]$} & $-29+\frac{1,990}{5,910} \cdot p$ \\
\hline
\end{tabular}

Table 1: Three different power bid price levels [Euro/MW] and energy bid price levels [Euro/MWh] for the positive German aFRR market (main period).

For the demand probability, an alternative approach for the identification of different price levels is required. Since the demand probability depends on the position in the energy bid merit-order which is determined by the value of the energy bid, two different functions need to be simulated:

- $x(p)$ - position in the merit-order of energy bids, and

- $n(x(p))$ - demand probability.

The function for the position in the energy bid merit-order needs to take into account the energy bid. In order to illustrate how different price levels can be modeled, energy bids for

\footnotetext{
${ }^{17}$ Note that the acceptance probability below (above) the lower (upper) bound of the power bid price level is one (zero).
} 
different positions in the merit-order of the energy bids are taken into account. Again, the positive market in the main period between January 2012 and December 2013 is considered. Table 2 shows the mean, minimum, and maximum energy bids $p$ that lead to position $x(p)$.

\begin{tabular}{ccccc}
$x(p)$ & $\operatorname{Mean}(p)$ & $\operatorname{Min}(p)$ & $\operatorname{Max}(p)$ & Std. Dev. $(p)$ \\
\hline 1 & 73 & 43 & 94 & 11 \\
100 & 80 & 59 & 118 & 13 \\
500 & 105 & 68 & 200 & 26 \\
1,000 & 161 & 83 & 415 & 61 \\
1,990 & 1,116 & 254 & 6,001 & 1,341 \\
\hline
\end{tabular}

Table 2: Energy prices [Euro/MWh] in the positive German aFRR market in the main period (regelleistung.net, 2017).

The energy bids substantially deviate at each position as illustrated by the values of the standard deviation Std. Dev. $(p)$. The standard deviation even increases with a higher position. This could be a hint to more hazardous bidding by suppliers: Since the demand probability decreases with a higher position, bidding high energy bids seems to be a tempting approach for generating high profits when being called.

For simplicity, only the lowest position $x(p)=1$ and the highest (for all demands recorded) position $x(p)=1990$ are considered for modeling different price levels. We assume a linear shape of $x(p)$ and refer to the minimum, mean and maximum values of the energy bids. Hence, three different price levels for the energy bid can be computed. Furthermore, for the sake of convenience, the values are rounded to the next decimal number. This is presented in the second part of Table 1.

We model the demand probability $n(x(p))$ as follows: Assuming that the demand for positive aFRR is denoted by $D$, that a linear shape can be applied and that the demand probability for positive and negative aFRR is the same (i.e., the maximum value is 0.5 ), the function is given by:

$$
n(x(p))=\frac{1}{2} \cdot\left(1-\frac{x(p)-1}{D-1}\right) .
$$


The lowest position in the energy bid merit-order is one, leading to a demand probability of 0.5. Hence, the position in the merit-order and the demand need to be corrected by one.

\section{Case Study: Numerical Application of Bidder's Decision- theoretic Calculus}

We compute optimal power and energy bids for different market scenarios subject to empirical based beliefs.

\section{Optimal Bidding Strategies}

We calculate the optimal power and energy bids for a fictive power plant. In order to carry out the calculation, we specify the market environment as well as certain characteristics of the power plant. That is, the power offer is set to $100 \mathrm{MW}$ and the variable costs to 20 Euro/MWh. For the sake of simplicity, it is assumed that all $100 \mathrm{MW}$ have the same position as the first MW, which leads to a slight exaggeration of the demand probability. Furthermore, the alternative market for the sale of energy has an expected price of 50 Euro/MWh. We consider the main period with a reserving period of 60 hours, and set the demand to 1,990 MW. Since the variable costs of the power plant are lower than the market price, the power plant is inframarginal and Operation Mode 1 is applied. Consequently, the capacity costs equal the opportunity costs of 1,800 Euro/MW.

To illustrate the effects that result from the variation of the power bid and energy bid price levels, we compute optimal power and energy bids for different market scenarios. With the help of the price levels from Section Empirical Identification of Acceptance and Demand Probability, we define nine different market scenarios. These and the corresponding optimal power and energy bids are presented in Table 3.

\section{Sample Calculation and Interpretation}

We provide a brief sample calculation of the profit for the case of both a low power bid and energy bid price level. Here, the optimal power bid is 115 Euro/MW which yields the 


\begin{tabular}{|c|c|c|c|c|}
\hline $\begin{array}{l}\text { Power bid } \\
\text { price level }\end{array}$ & $\begin{array}{l}\text { Energy bid } \\
\text { price level }\end{array}$ & $\begin{array}{c}b^{*} \\
{[\text { Euro/MW] }}\end{array}$ & $\begin{array}{c}p^{*} \\
{[\text { Euro/MWh] }}\end{array}$ & $\begin{array}{c}\text { Expected profit } \\
{[\text { Euro }]}\end{array}$ \\
\hline \multirow{3}{*}{ Low } & Low & 115 & 135 & $\approx 13 \mathrm{k}$ \\
\hline & Medium & 0 & 550 & $\approx 600 \mathrm{k}$ \\
\hline & High & 0 & 3,010 & $\approx 4,355 \mathrm{k}$ \\
\hline \multirow{3}{*}{ Medium } & Low & 315 & 135 & $\approx 22 \mathrm{k}$ \\
\hline & Medium & 0 & 550 & $\approx 600 \mathrm{k}$ \\
\hline & High & 0 & 3,010 & $\approx 4,355 \mathrm{k}$ \\
\hline \multirow{3}{*}{ High } & Low & 505 & 135 & $\approx 38 \mathrm{k}$ \\
\hline & Medium & 160 & 550 & $\approx 700 \mathrm{k}$ \\
\hline & High & 160 & 3,010 & $\approx 4,371 \mathrm{k}$ \\
\hline
\end{tabular}

Table 3: Different market scenarios in the German positive aFRR market (main period) and optimal power and energy bids.

following profit of the power bid per MW (see term (4)):

$$
\pi_{P}(b) / q=(115-1,800) \text { Euro/MW }=-1,685 \text { Euro/MW. }
$$

The subjective acceptance probability is around $64.1 \%$. The optimal energy bid is 135 Euro/MWh which leads an expected position in the merit-order of $x(p)=899$. At this position, the subjective demand probability is around $n(x(p))=27.4 \%$ of the 60 hours in the main period, i.e., approximately 16.44 hours. Consequently, the expected profit of the energy bid per MW is given by (see term (6)):

$$
\pi_{E}^{e}(p)=(135-20) \text { Euro/MWh } \cdot 16.44 \mathrm{~h} \approx 1,891 \text { Euro/MW. }
$$

Adding up the profits of the power bid per MW and the expected profits of the energy bids per MW yields combined profits of 206 Euro/MW.

Finally, the overall expected profit results in (see term (7)):

$$
\pi^{e}(b, p)=64.1 \% \cdot 206 \text { Euro/MW } \cdot 100 \mathrm{MW} \approx 13 k \text { Euro. }
$$

The expected profits for all scenarios are positive, i.e., any supplier with the given characteristics participates at the aFRR auction. The expected profit is rising (or at the same 
level) with a higher energy bid price level (power bid price level) at a fixed power bid price level (energy bid price level). The lowest (highest) expected profit is reached at a low (high) power bid and low (high) energy bid price level.

The optimal energy bids are the same for each energy bid price level, independent of the power bid price level. This reflects the reasoning in Section Optimal Power and Energy Bid: The optimal energy bid is independent of the power bid. Besides, the optimal energy bids are (far) higher than the variable costs. The reason for this is that the respective expected energy price levels are considered when calculating the energy bid.

On the contrary, the optimal power bids differ with respect to the three energy price levels. Although the optimal power bids are continuously lower than the opportunity costs, bidding the lower boundary of the power bid price level is maximizing the expected profit if a medium or high energy bid price level is expected. Why is that? On the one hand, bidding the lower boundary of the expected power bid price level yields a high probability of acceptance for the power bid. ${ }^{18}$ Since the profits of the energy bid in the medium and high energy bid price level are extremely large, possible profits of the power bid are neglected. This goes hand in hand with the results from Section Optimal Power and Energy Bids: Expected profits of the energy bid are taken into account for the calculation of the optimal power bid. On the other hand, opportunity costs in this example are small with respect to the medium and high energy price level.

\section{Validation of Theoretic Results with Empirical Auction Data}

A central result of our decision-theoretic approach is that the power and energy bids are not independent of each other, i.e., expected profits of the energy bid are taken into account for the calculation of the optimal power bid. This holds for both the positive and negative aFRR markets and is in line with recent scientific work (Müsgens et al., 2014; Ocker \& Ehrhart, 2017a). As Section Case Study: Numerical Application of Bidder's Decision-theoretic Calculus shows, this can lead to extreme optimal bidding behavior, i.e., setting the power bid

\footnotetext{
${ }^{18}$ The tie-breaking rule is based on the power bid, then the energy bid, and finally on the size of the power offer (regelleistung.net, 2018; Austrian Power Grid, 2018).
} 
to the lower boundary of the expected power bid price if the expected energy bids are high.

To validate our theoretic findings, we use empirical aFRR market data from Austria and Germany. In Figures 7 and 8, the auction results for the German and Austrian positive and negative aFRR market are depicted for the time period from January 2014 to May 2016 (regelleistung.net, 2018; Austrian Power Grid, 2018). ${ }^{19}$ Note that in the following the auction results for the power bid are given in Euro/MWh, i.e., a separate average power bid for each hour is stated. We selected this form of representation since it enables to depict the main period (Haupttarif, HT) and sub-period (Nebentarif, NT) in one figure.

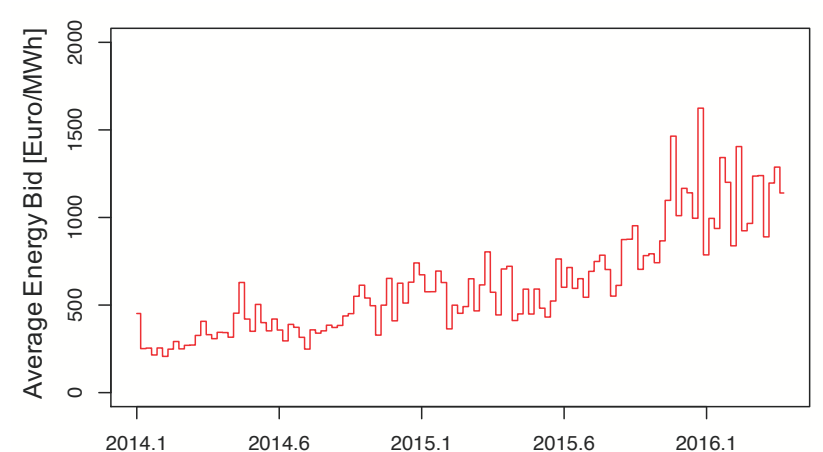

Positive market (HT and NT combined)

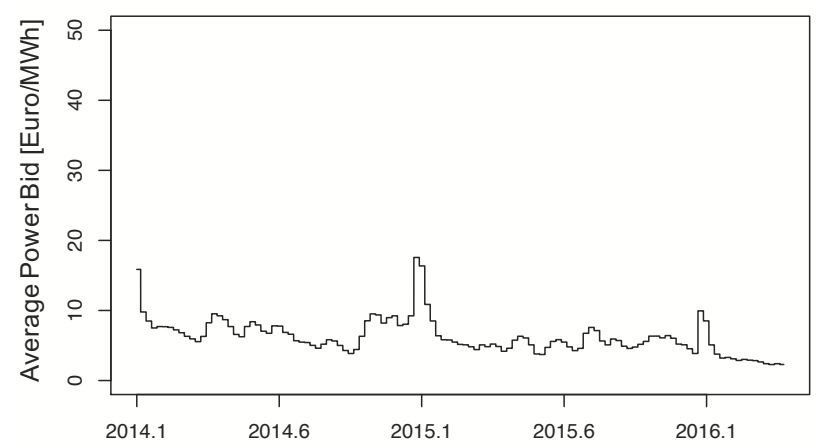

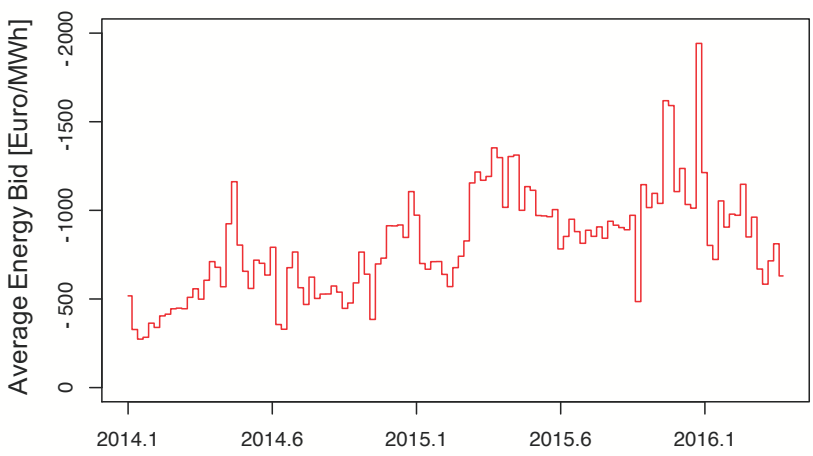

Negative market (HT and NT combined)

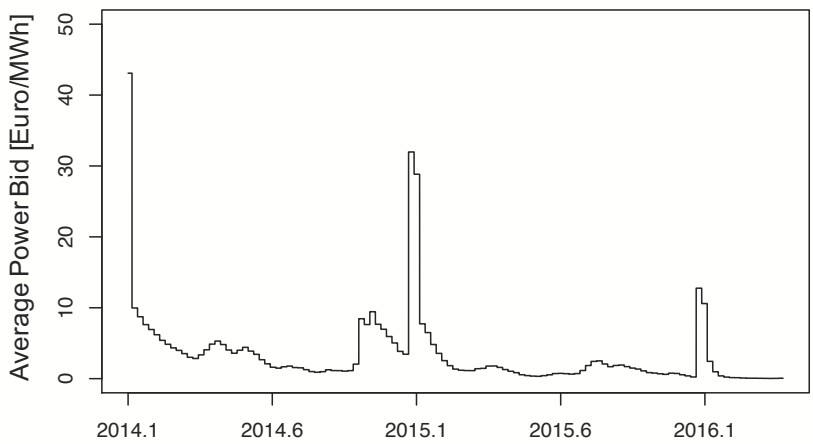

Figure 7: Empirical market data of the positive and negative German aFRR market in the time period from January 2014 to May 2016; main and sub-period combined; power bid converted to Euro/MWh; source: regelleistung.net (2017).

We find that our theoretic results are in line with empirical auction data from Austria and Germany. The range of the optimal power and energy bids identified in the numerical

\footnotetext{
${ }^{19}$ We chose this time period for our main empirical analysis because, beginning with July 2016, the Austrian and German aFRR markets merged. For an analysis of the prices after the merger see the end of this section.
} 

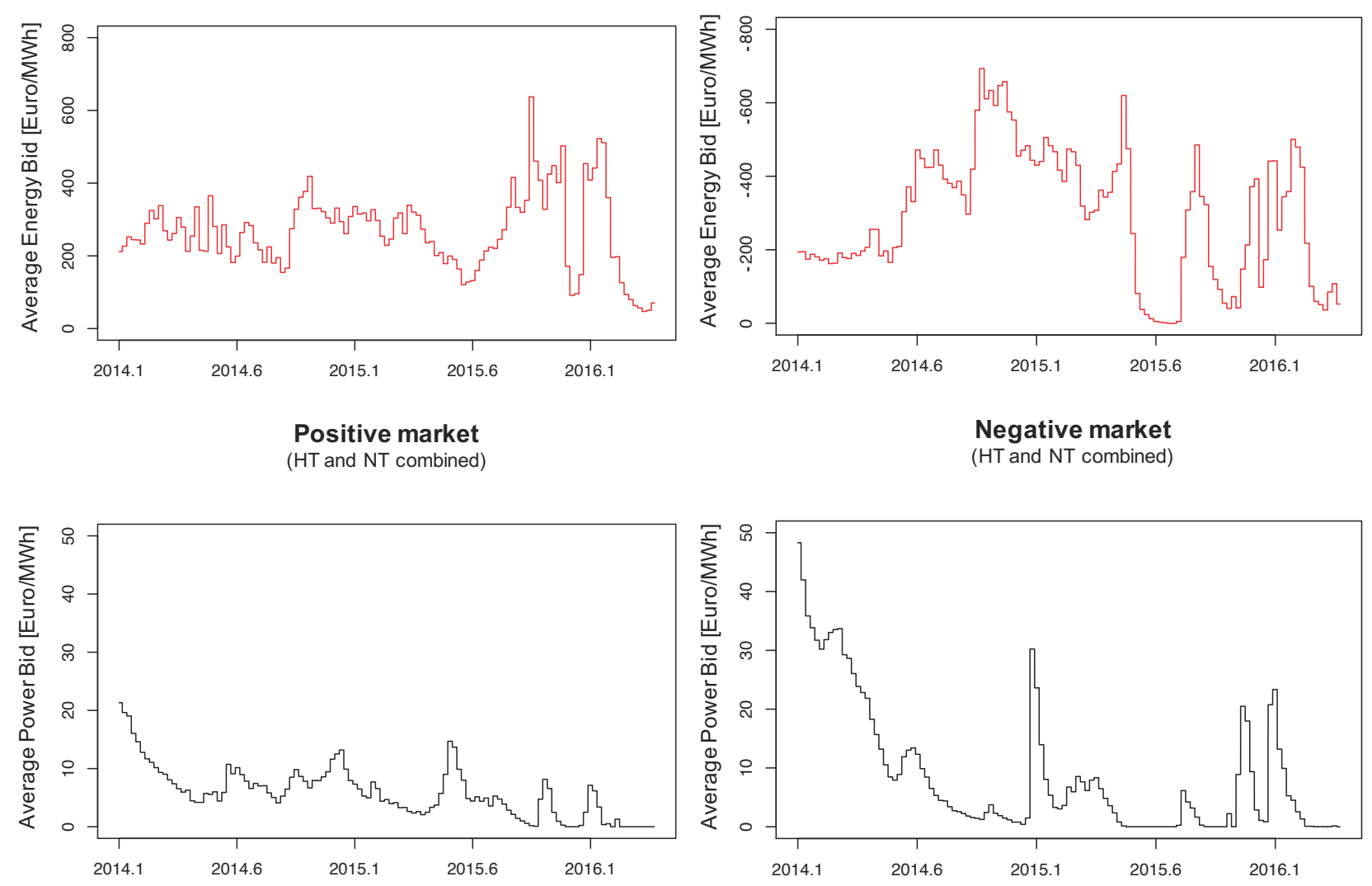

Figure 8: Empirical market data of the positive and negative Austrian aFRR market in the time period from January 2014 to May 2016; main and sub-period combined; power bid converted to Euro/MWh; source: Austrian Power Grid (2017).

example in Section Case Study: Numerical Application of Bidder's Decision-theoretic Calculus is reflected in the German market data. ${ }^{20}$ Moreover, the inverse relationship of the two bid components is unambiguous: Increasing energy bid prices come along with decreasing power bid prices. ${ }^{21}$ Testing for Spearman's rank correlation confirms the hypothesized negative relationship as statistically significant in both German aFRR markets. ${ }^{22}$ In the

\footnotetext{
${ }^{20}$ Recall that the numerical example in Section Case Study: Numerical Application of Bidder's Decisiontheoretic Calculus used market data from Germany. Furthermore, the average power bids in the figures are given in Euro/MW, i.e., when comparing the values, the identified optimal power bids of the numerical example need to be divided by 60 .

${ }^{21}$ Only the time span around Christmas and New Year's Eve contradicts this trend. However, this can be traced back to an increased demand for balancing power and thus higher prices (Ocker \& Ehrhart, 2017a).

${ }^{22}$ One-tailed correlation test (Spearman's rank correlation $\rho$ ), $n=19,895: \rho=-0.42$, p-value $<0.001$ (positive aFRR market); $\rho=-0.37, \mathrm{p}$-value $<0.001$ (negative aFRR market).
} 
Austrian aFRR markets, the identified negative correlation of the power and energy bid is also confirmed in the negative aFRR market. ${ }^{23}$

Furthermore, even the identified extreme bidding behavior is reflected in the empirical market outcomes. In the considered time period, $32 \%$ (19\%) of the average power bids in the negative (positive) Austrian aFRR market, and $36 \%(0 \%)$ of the average power bids in the negative (positive) German aFRR are lower than 1 Euro/MWh (the lowest possible value for the power bid is zero). The higher share of average power bids that are lower than 1 Euro/MWh in the two negative aFRR markets can be traced back to the underlying cost structures, since there are no opportunity costs that need to be reflected in the power bids in the case of inframarginal power plants for the negative market (see Section Capacity Costs).

Since July 2016, the Austrian and German aFRR markets merged, i.e., there exists a common procurement for the merit-order. According to the Austrian and German TSOs, the market merger serves as a trailblazer for the future harmonization of the European aFRR markets, e.g., it lowers the costs for balancing energy because the cheapest aFRR in both countries can be utilized (regelleistung.net, 2018; Austrian Power Grid, 2018).

We also analyze market data after the cooperation and focus on the energy prices on high merit-order positions in the positive market. Figure 9 depicts the energy bids on position 1,900 in the German merit-order from January 2016 to June 2016 and in the common meritorder from July 2016 to August 2017. The development is clearly influenced by the common procurement of balancing energy: there is a substantial decline in prices at the start of the cooperation. However, prices did not remain at this low level but increased again over time. A reason for this may be the limited grid transmission capacity between Austria and Germany: if these transmission capacities are exhausted, the common merit-order is split again, leading to two separated markets. In this case, the suppliers face a reduced competition and, thus, are able to establish high energy bid price levels.

The German power bid prices reacted as predicted on the development of the energy prices: At the beginning of the cooperation, the power bid prices were stable at around five Euro/MWh, but seem to converge to zero since January 2017 (regelleistung.net, 2018).

\footnotetext{
${ }^{23}$ One-tailed correlation test (Spearman's rank correlation $\rho$ ), $n=19,895: \rho=0.07$, p-value $>0.5$ (positive aFRR market); $\rho=-0.19, \mathrm{p}$-value $<0.001$ (negative aFRR market).
} 


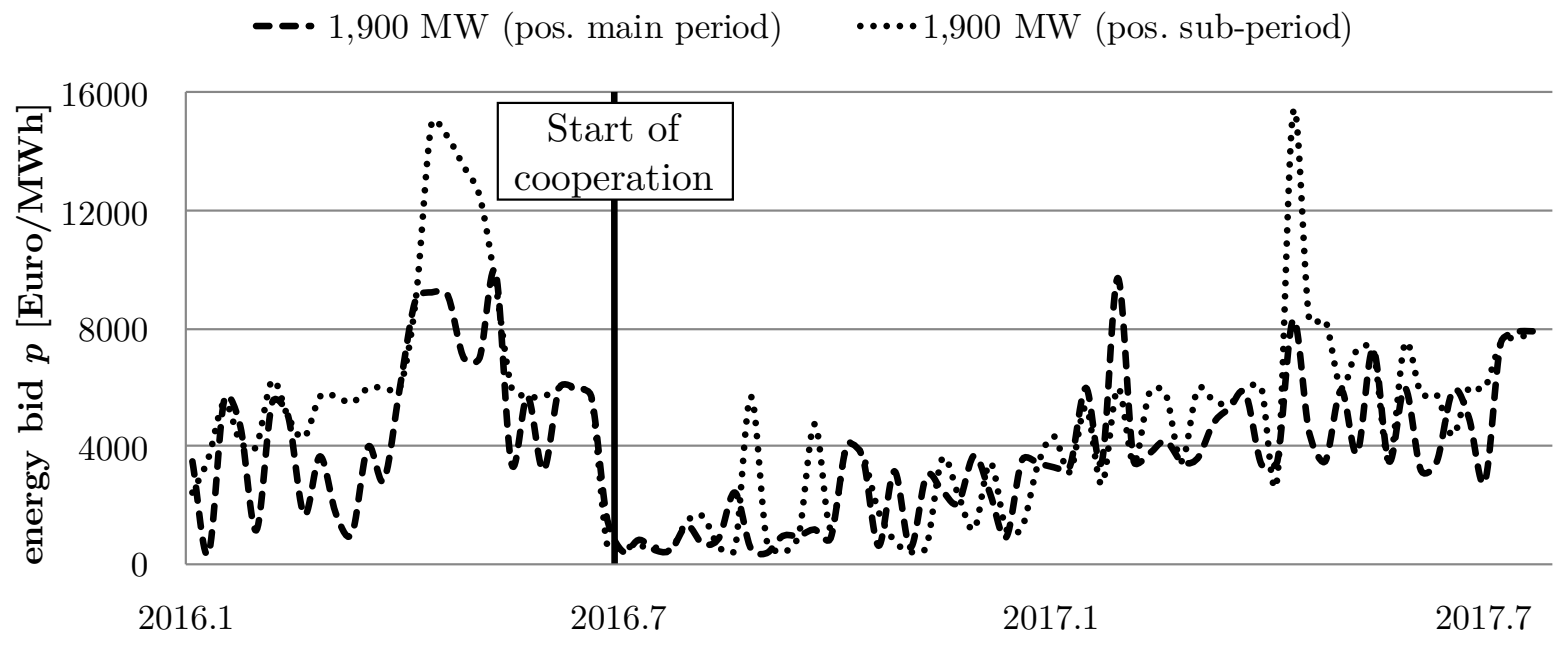

Figure 9: Merit-order position 1,900 MW in the German merit-order from 2016.1 to 2016.6 and in the common merit-order from 2016.7 to 2017.8 for the positive Austrian-German aFRR market (main period and sub-period); source: regelleistung.net (2017).

\section{CONCLUSIONS}

This paper presents an economic analysis of bidding strategies in the Austrian and German aFRR markets. Based on the analysis of the operating principle, the underlying cost and profit structures, we develop a bidder's decision-theoretic calculus that leads to optimal power and energy bids, allowing the integration of price expectations.

A central result of our approach is that the two bids are not independent of each other, i.e., expected profits of the energy bid are taken into account for the calculation of the optimal power bid. Our numerical example of the bidder's calculus illustrates this result. If high energy bid prices can be expected, the representative supplier bids a low power bid. The reason for this is the applied scoring rule which consists only of the power bid: Bidding a low power bid increases the probability of being awarded. Hence, in this case, the power bid is subsidized: The representative supplier understates its true costs for keeping balancing power available to the grid. The low power bid is in return compensated with an (extremely) high energy bid. In contrast, if the price level for the energy bids is low, the representative supplier increases her power bids conversely. We define different market scenarios by analyzing the development of the market prices, and present a case study of 
the bidder's calculus for three scenarios. Finally, we validate our theoretic findings with empirical auction data from Austria and Germany. We find that the negative correlation of the two bid components is confirmed in both the Austrian and German aFRR markets.

These findings are of relevance regarding the intended European-wide harmonization of the aFRR auction, since the future market shell build mostly on the Austrian and German auction design (European Commission, 2017). Based on the findings of this paper, we strongly encourage the European Commission to take into account empirically observed bidding strategies when designing a common auction. Our results point towards coordinated bids based on previous auction outcomes. Therefore, we appreciate the planned introduction of voluntary energy bids. Here, suppliers who were not awarded or did not participate in the regular auction have the opportunity to submit a second, exclusive energy bid in order to be part of the merit-order of energy bids. Voluntary energy bids were already implemented in Austria for wind power plants and in the Netherlands for all types of plants. The analysis of auction results in the Netherlands indicates that these bids foster a competitive price level for energy bids (Ocker, 2017). Therefore, we consider the implementation of voluntary energy bids as a first step to enhance competition and impede collusive behavior, which is facilitated by the regular repetition of the auctions (Belica, Ehrhart, \& Ocker, 2016) and the limited set of suppliers (Knaut, Obermüller, \& Weiser, 2017).

Further research could develop an integrated model for both the wholesale and balancing power market. Here, the interdependencies between the wholesale energy price and the balancing power price can be investigated more rigorously. This includes especially the overall efficiency in both markets.

\section{References}

Asker, J., \& Cantillon, E. (2008). Properties of scoring auctions. The RAND Journal of Economics, 39(1), 69-85. (doi: https://doi.org/10.1111/j.1756-2171.2008.00004.x)

Asker, J., \& Cantillon, E. (2010). Procurement when price and quality matter. The RAND Journal of Economics, 41(1), 1-34. (doi: https://doi.org/10.1111/j.17562171.2009.00088.x) 
Austrian Power Grid. (2018). Auction results. [accessed 27-February-2018]. Retrieved from https ://www .apg . at

Ausubel, L. M., Cramton, P., Pycia, M., Rostek, M., \& Weretka, M. (2014). Demand reduction and inefficiency in multi-unit auctions. The Review of Economic Studies, 81, 1366-1400. (doi: https://doi.org/10.1093/restud/rdu023)

Belica, M., Ehrhart, K.-M., \& Ocker, F. (2016). Kritische Beurteilung der geplanten Umstrukturierung des Regelenergiemarktes. Energiewirtschaftliche Tagesfragen, 5, 13-14.

Bergemann, D., \& Wambach, A. (2015). Sequential information disclosure in auctions. Journal of Mathematical Economics, 159, 1074-1095. (doi: https://doi.org/10.1016/j.jet.2014.11.004)

Bessa, R., Moreira, C., Silva, B., \& Matos, M. (2014). Handling renewable energy variability and uncertainty in power systems operation. Wiley Interdisciplinary Reviews Energy and Environment, 3(2), 156-178. (doi: https://doi.org/10.1002/wene.76)

Bichler, M., \& Kalagnanam, J. (2005). Configurable offers and winner determination in multi-attribute auctions. European Journal of Operational Research, 160(2), 380-394. (doi: https://doi.org/10.1016/j.ejor.2003.07.014)

Branco, F. (1997). The design of multidimensional auctions. The RAND Journal of Economics, 28(1), 63-81. (doi: https://doi.org/10.2307/2555940)

Bushnell, J. B., \& Oren, S. S. (1995). Bidder cost revelation in electric power auctions. Journal of Regulatory Economics, 6(1), 5-26. (doi: https://doi.org/10.1007/BF01065387)

Chao, H.-P., \& Wilson, R. (2002). Multi-dimensional procurement auctions for power reserves: Robust incentive-compatible scoring and settlement rules. Journal of Regulatory Economics, 22(2), 161-183. (doi: https://doi.org/10.1023/A:1020535511537)

Che, Y. K. (1993). Design competition through multidimensional auctions. The RAND Journal of Economics, 24(4), 668-680. (doi: https://doi.org/10.2307/2555752)

Cramton, P., \& Ockenfels, A. (2012). Economics and design of capacity markets for the power sector. Zeitschrift für Energiewirtschaft, 36, 113-134. (doi: https://doi.org/10.1007/s12398-012-0084-2)

Cramton, P., \& Schwartz, J. (2000). Collusive bidding: Lessons from the FCC spectrum auctions. Journal of Regulatory Economics, 17(3), 229-252. (doi: 
https://doi.org/10.1023/A:1008174031940)

David, E., Azoulay-Schwartz, R., \& Kraus, S. (2006). Bidding in sealed-bid and english multi-attribute auctions. Decision Support Systems, 42(2), 527-556. (doi: https://doi.org/10.1016/j.dss.2005.02.007)

Ehrhart, K.-M. (2001). European central bank operations: experimental investigation of the fixed rate tender. Journal of International Money and Finance, 20(6), 871-893. (doi: https://doi.org/10.1016/S0261-5606(01)00027-4)

Ellison, J. F., Tesfatsion, L. S., Loose, V. W., \& Byrne, R. H. (2012). Project report: A survey of operating reserve markets in u.s. iso/rto-managed electric energy regions. [accessed 19-March-2018], 1-41. Retrieved from http://www.sandia.gov/ess/ publications/SAND2012_1000.pdf

ENTSO-E. (2017). Network codes electricity balancing. [accessed 13-December-2017]. Retrieved from https://electricity.network-codes.eu/network_codes/eb/

European Commission. (2017). Electricity balancing guideline. Commission Regulation (EU) 2017/2195 of 23 November 2017 establishing a guideline on electricity balancing [accessed 13-December-2017]. Retrieved from http://eur-lex.europa.eu/ legal-content/EN/TXT/?uri=CELEX : 32017R2195

Gretschko, V., Rasch, A., \& Wambach, A. (2014). On the strictly descending multi-unit auction. Journal of Mathematical Economics, 50, 79-85. (doi: https://doi.org/10.1016/j.jmateco.2013.10.009)

Heim, S., \& Götz, G. (2013). Do pay-as-bid auctions favor collusion? Evidence from Germany's market for reserve power. ZEW - Centre for European Economic Research Discussion Paper No. 35..

Herbsman, Z. J., Chen, W. T., \& Epstein, W. C. (1995). Time is money: innovative contracting methods in highway construction. Journal of Construction Engineering and Management, 121(3), 273-281. (doi: https://doi.org/10.1061/(ASCE)07339364(1995)121:3(273))

Hirth, L., \& Ziegenhagen, I. (2015). Balancing power and variable renewables: Three links. Renewable and Sustainable Energy Reviews, 50, 1035-1051. (doi: https://doi.org/10.1016/j.rser.2015.04.180) 
Holttinen, H. (2012). Wind integration: experience, issues, and challenges. Wiley Interdisciplinary Reviews Energy and Environment, 1(3), 243-255. (doi: https://doi.org/10.1002/wene.18)

Hortacsu, A., \& Puller, S. L. (2008). Understanding strategic bidding in multi-unit auctions: a case study of the texas electricity spot market. The RAND Journal of Economics, 39(1), 86-114. (doi: https://doi.org/10.1111/j.0741-6261.2008.00005.x)

Ishii, R. (2014). Bid roundness under collusion in Japanese procurement auctions. Review of Industrial Organization, 44 (3), 241-254. (doi: https://doi.org/10.1007/s11151-013$9408-6)$

Just, S., \& Weber, C. (2008). Pricing of reserves: Valuing system reserve capacity against spot prices in electricity markets. Energy Economics, 30, 3198-3221. (doi: https://doi.org/10.1016/j.eneco.2008.05.004)

Kahn, A. E., Cramton, P. C., Porter, R. H., \& Tabors, R. D. (2001). Uniform pricing or pay-as-bid pricing: A dilemma for California and beyond. The Electricity Journal, 14(6), 70-79. (accessible via ftp://cramton.umd.edu/papers2000-2004/kahn-cramtonporter-tabors-uniform-or-pay-as-bid-pricing-ej.pdf)

Kamat, R., \& Oren, S. S. (2002). Rational buyer meets rational seller: Reserves market equilibria under alternative auction designs. Journal of Regulatory Economics, 21(3), 247-288. (doi: https://doi.org/10.1023/A:1015378914995)

Knaut, A., Obermüller, F., \& Weiser, F. (2017). Tender frequency and market concentration in balancing power markets. Institute of Energy Economics at the University of Cologne (EWI) Working Paper, 17/04, 242-259. (accessible via http://www.ewi.unikoeln.de/fileadmin/user_upload/Publikationen/Working_Paper/EWI_

_WP_17_04_Tender_Frequency_and_Market_Concentration.pdf)

Kremer, I., \& Nyborg, K. G. (2009). Underpricing and market power in uniform price auctions. The Review of Financial Studies, 17(3), 849-877. (doi: https://doi.org/10.1093/rfs/hhg051)

Lu, Y., Gupta, A., Ketter, W., \& Heck, E. V. (2014). Information transperancy in multichannel B2B auctions: A field experiment. Thirty Fourth International Conference on Information Systems,. ( accessible via http://citeseerx.ist.psu.edu/viewdoc/download? 


$$
\text { doi }=10.1 \cdot 1.883 .6777 \& \text { rep=rep1\&type=pdf) }
$$

Macatangay, R. E. (2002). Tacit collusion in the frequently repeated multi-unit uniform price auction for wholesale electricity in England and Wales. European Journal of Law and Economics, 13(3), 257-273. (doi: https://doi.org/10.1023/A:1014730803412)

McAfee, R. P. (1993). Mechanism design by competing sellers. Econometrica, 61(6), 12811312. (doi: https://doi.org/10.2307/2951643)

Milgrom, P. R., \& Weber, R. J. (1982). A theory of auctions and competitive bidding. Econometrica, 50(5), 1089-1122. (doi: https://doi.org/10.2307/1911865)

Müller, G., \& Rammerstorfer, M. (2008). A theoretical analysis of procurement auctions for tertiary control in Germany. Energy Policy, 36, 2620-2627. (doi: https://doi.org/10.1016/j.enpol.2008.03.017)

Müsgens, F., Ockenfels, A., \& Peek, M. (2014). Economics and design of balancing power markets in Germany. International Journal of Electrical Power 83 Energy Systems, 55, 392-401. (doi: https://doi.org/10.1016/j.ijepes.2013.09.020)

Myerson, R. B. (1981). Optimal auction design. Mathematics of operations research, 6(1), 58-73. (stable URL: http://links.jstor.org/sici?sici=0364765X\%28198102\%296\%3A1\%3C58\%3AOAD\%3E2.0.CO\%3B2-C)

Ocker, F. (2017). Design and performance of European balancing power auctions. Proceedings 14th International Conference on the European Energy Market, 1-6. (doi: https://doi.org/10.1109/EEM.2017.7981861)

Ocker, F., Braun, S., \& Will, C. (2016). Design of European balancing power markets. Proceedings of the 13th International Conference on the European Energy Market, 1-6. (doi: https://doi.org/10.1109/EEM.2016.7521193)

Ocker, F., \& Ehrhart, K.-M. (2017a). The "German paradox" in the balancing power markets. Renewable and Sustainable Energy Reviews, 67, 892-898. (doi: https://doi.org/10.1016/j.rser.2016.09.040)

Ocker, F., \& Ehrhart, K.-M. (2017b). Short-term turnaround for the procurement of new power plants for the german grid reserve: An economic analysis. Zeitschrift für Energiewirtschaft, 41, 23-32. (doi: https://doi.org/10.1007/s12398-016-0188-1)

Ocker, F., Ehrhart, K.-M., \& Belica, M. (2018). Harmonization of the European balancing 
power auction: A game-theoretical and empirical investigation. Working Paper KIT Karlsruhe.

Rammerstorfer, M., \& Wagner, C. (2009). Reforming minute reserve policy in Germany: A step towards efficient markets? Energy Policy, 37, 3513-3519. (doi: https://doi.org/10.1016/j.enpol.2009.03.056)

regelleistung.net. (2018). Internetplattform zur Vergabe von Regelleistung. [accessed 27February-2018]. Retrieved from https://www.regelleistung.net

Schweizer, S., \& Mattis, M. (2016). Die neuen gesetzlichen instrumente zur versorgungssicherheit im deutschen stromnetz. Energiewirtschaftliche Tagesfragen, 5, 84-89.

Skrzypacz, A., \& Hopenhayn, H. (2004). Tacit collusion in repeated auctions. Journal of Economic Theory, 114(1), 153-169. (doi: https://doi.org/10.1016/S0022-0531(03)001285)

Söder, L. (2016). Simplified analysis of balancing challenges in sustainable and smart energy systems with 100\% renewable power supply. Wiley Interdisciplinary Reviews Energy and Environment, 5(4), 401-412. (doi: https://doi.org/10.1002/wene.194)

Swider, D. J., \& Weber, J. (2007). Bidding under price uncertainty in multi-unit pay-asbid procurement auctions for power systems reserve. European Journal of Operational Research, 181, 1297-1308. (doi: https://doi.org/10.1016/j.ejor.2005.11.046)

Wen, F., \& David, A. (2002). Coordination of bidding strategies in day-ahead energy and spinning reserve markets. International Journal of Electrical Power $\mathcal{E}$ Energy Systems, 24(4), 251-261. (doi: https://doi.org/10.1016/S0142-0615(01)00038-2)

Wolfram, C. D. (1997). Strategic bidding in a multi-unit auction: An empirical analysis of bids to supply electricity (Tech. Rep.). National Bureau of Economic Research. 